\title{
Exploratory Study on Digital transformation: Capabilities and Expected Performances
}

\author{
Byungchul Choi ${ }^{1,{ }^{*}}$ and Seunghyun Kim ${ }^{2}$ \\ ${ }^{1}$ College of Business, Hankuk University of Foreign Studies, Seoul, South Korea \\ ${ }^{2}$ Science and Technology Policy Institute, Sejong, South Korea
}

\begin{abstract}
Nowadays many firms encounter the macro-level changes called digital transformation and research on it has drastically increased since 2014. In response to this emerging phenomenon, this study explores how firms prepare for digital transformation and what do they expect from it. More specially, based on the survey from 439 Korean scaleup firm (high-growth firm), we suggest the concepts to necessary capabilities for and expected performance improvement through digital transformation. Our study illustrates that how a firm's perceptions on those concepts varies upon firm size and industry type. Additionally, our study offers a clue for 'digital divide' that can potentially threaten the survival of numerous firms.
\end{abstract}

Keywords: digital transformation, capabilities, performance improvement, firm size, industry type

\section{Introduction}

In recent years, the business world has encountered the macro-level changes sourced from of digital transformation. While digital transformation is not a completely new topic to many entities across the areas, the growing body of research has been shown since 2014 (see Figure 1, Google trend). However, as mentioned above digital transformation comes with unprecedented changes and convergence across various technological domain and industrial sectors, it can be a double-edged sword to both incumbents and new ventures. Although it can potentially provide diverse and constructive collaboration opportunities to many firms (Luo, 2021), it can also threat the business of many firms as existing technological standards and business systems will be drastically either changed or challenged (Oh \& Rhee, 2008).

In response to this mixed expectation on digital transformation, this study explore how firms are currently viewing digital transformation in terms of preparation and desirable outcomes. More specifically, by using the Korean high-growth firm data, we first offer the results of survey implying what types of capabilities those high-growth firms pereceive as key capabilities to cope with changes sourced from digital transformation and what types of firm performances they anticipate to achieve. Then, we shift our attention to important firmspecific characteristics, firm size and industry type that can matter when firm adopt marcolevel changes (Li et al., 2018; Eller et al., 2020; Siachou et al., 2021; Savastano et al., 2019;

${ }^{*}$ Corresponding author: bchoi@hufs.ac.kr 
Vogelsang et al., 2018; von Leipzig et al., 2017; Martín-Peña et al., 2020; Ribeiro-Navarrete et al., 2021), and provides a comparative analysis. The results indicate taht firms' perception of and preparations for digital transformation vary upon firm-specific charateristics. We also find that some firms might suffer from digital divide (bipolarization of digital capability).

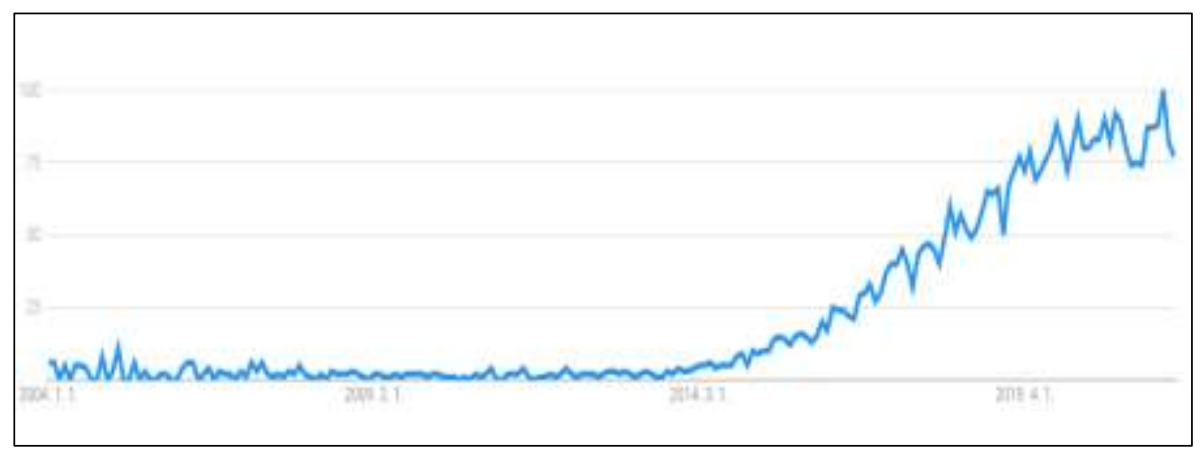

Fig. 1. Interest in Digital Transformation

Source: Google Trend.

\section{Research Design}

\subsection{Selection of capabilities and performance}

To design survey, we select the following three capabilities: (1) business capability that refers to a firm's ability for task management, process improvement, and data management (2) digital capability, which is defined as a firm's technological capability for programming, software development, and data analysis (3) soft capability that refers to a firm's capability for problem-solving, collaboration, and creativity management. Those capabilities are necessary to achieve innovation (Teece, 1986, 2018). The reason we focus on these three capabilities is that a firm's ability to cope with macro-level changes ultimately depends on how a firm develop necessary technologies, how a firm apply those technologies to real business activities and how a people in those firms manage these transitional processes. Regarding anticipated performances that firms eventually aim to achieve, we develop three performance dimensions, which are (1) profitability growth (2) new product development and (3) new partnership.

\subsection{Sample collection}

We implemented a survey based on the list of scaleup firms in Korea. To collect the sample firms, we first draw on the Korea Enterprise Data (KED) database subscribed by Science and Technology Policy Institute (STEPI), and gather the initial sample firms that have shown 20 percent increase in sales with more than 10 employees during the period of 2016-2018. This initial sample include 3,391 scaleup firms. Then, we sent email survey to all these firms and a total of 439 survey was received with response rate of $13 \%$. We classified those 439 firms based on two categories, firm size and industries. Regarding the former category, while 86 firms (20\%) belong to a large firm, 353 firms (80\%) were Small and Medium Enterprises (SMEs). In relation the latter category, while manufacturing industries comprised $44 \%$ of the sample (194 firms), non-manufacturing firms such as service consist 56\% of the sample (245 
firms). We designed a survey questionnaire by referring to 2019 ScaleUp Survey of Scaleup Institute, the UK private sector, not-for-profit company (s).

\subsection{Analysis}

Collected data were analyzed with a focus on the following four matters: (1) importance of digital transformation and preparations, (2) expected change digital transfor-mation would bring to the industry and main products, (3) capabilities necessary to prepare for digital transformation, and (4) anticipated performance improvement from digi-tal transformation. Digital transformation is a macro-trend phenomenon that affects all industries and many firms, but individual firms may have different views depending on their characteristics or the industry to which they belong. Therefore, this study applies the classification method according to firm size and industrial characteristics for each of the aforementioned matters.

\section{Results}

Table 1 shows firms' percption on "capabilities considered important to prepare digital transformation" depending on firm size and industry typel. In all capabilty types, smallmedium size firms show more than $50 \%$ o "neutral" views and low level of 'Positive' views, indicating that small and medicum size firms have pessimistic perspective on needs of those capabilites. Simiarily, compare to manufaturing firms, non-manufacturing firms have more 'Neutral' and 'Negative' perspective on the value of each capability.

Table 1. Do you think this capability is important to prepare digital transformation?

\begin{tabular}{|c|c|c|c|c|c|}
\hline & & \multicolumn{4}{|c|}{ Business capability (\%) } \\
\hline & & $\mathrm{N}$ & Negative & Neutral & Positive \\
\hline Category & Large & 86 & 5.9 & 43.0 & 51.2 \\
\hline 1 & Small-Medium & 353 & 18.1 & 51.6 & 30.3 \\
\hline \multirow[t]{4}{*}{ Category 2} & Manufacturing & 194 & 12.4 & 51.0 & 36.6 \\
\hline & Non-Manufacturing & 245 & 23.2 & 61.9 & 14.9 \\
\hline & & & \multicolumn{3}{|c|}{ Digital capability (\%) } \\
\hline & & $\mathrm{N}$ & Negative & Neutral & Positive \\
\hline Category & Large & 86 & 5.9 & 44.2 & 50 \\
\hline 1 & Small-Medium & 353 & 22.7 & 50.1 & 27.1 \\
\hline \multirow[t]{4}{*}{ Category 2} & Manufacturing & 194 & 14.4 & 52.6 & 32.9 \\
\hline & Non-Manufacturing $\mathrm{f}$ & 245 & 29.4 & 58.2 & 12.4 \\
\hline & & & \multicolumn{3}{|c|}{ Soft capability (\%) } \\
\hline & & $\mathrm{N}$ & Negative & Neutral & Positive \\
\hline Category & Large & 86 & 7 & 43.0 & 50 \\
\hline 1 & Small-Medium & 353 & 19.9 & 51.8 & 28.3 \\
\hline \multirow[t]{2}{*}{ Category 2} & Manufacturing & 194 & 13.4 & 53.6 & 33 \\
\hline & Non-Manufacturing & 245 & 25.7 & 59.8 & 14.5 \\
\hline
\end{tabular}

Source: Own processing

Table 2 shows firms' expectation on 'performance impovement through digital transformation. Compared to small-medium firms, large firms show more optimistic view and less pessemistic view on each performance dimenstion. Similarily, compared to nonmanufacuting firms, manufacturing firms show more optimistic view on each performance dimenstion. 
Table 2. What types of performance you want to improve through digital transformation?

\begin{tabular}{|c|c|c|c|c|c|}
\hline & & \multicolumn{4}{|c|}{ Profitability growth (\%) } \\
\hline & & $\mathrm{N}$ & Pessemistic & Neutral & Optimistic \\
\hline Category & Large & 86 & 24.5 & 54.7 & 20.9 \\
\hline 1 & Small-Medium & 353 & 54.1 & 35.7 & 10.2 \\
\hline Category & Manufacturing & 194 & 45.8 & 38.7 & 15.5 \\
\hline \multirow[t]{3}{*}{2} & Non-Manufacturing & 245 & 37.1 & 50.5 & 12.4 \\
\hline & & \multicolumn{4}{|c|}{ New product development (\%) } \\
\hline & & $\mathrm{N}$ & Pessemistic & Neutral & Optimistic \\
\hline Category & Large & 86 & 25.6 & 59.3 & 15.1 \\
\hline 1 & Small-Medium & 353 & 55.8 & 36.5 & 7.6 \\
\hline Category & Manufacturing & 194 & 45.9 & 41.8 & 12.4 \\
\hline \multirow[t]{3}{*}{2} & Non-Manufacturing & 245 & 40.7 & 51.0 & 8.3 \\
\hline & & \multicolumn{4}{|c|}{ New partnership (\%) } \\
\hline & & $\mathrm{N}$ & Pessemistic & Neutral & Optimistic \\
\hline Category & Large & 86 & 26.7 & 55.8 & 17.4 \\
\hline 1 & Small-Medium & 353 & 56 & 37.1 & 6.8 \\
\hline Category & Manufacturing & 194 & 47.4 & 40.2 & 12.3 \\
\hline 2 & Non-Manufacturing & 245 & 40.1 & 52.1 & 7.8 \\
\hline
\end{tabular}

Source: Own processing

After explore the general perspective of firms on each dimenion of capability and perforamnce, we conduct sets of OLS regression to get further insights on the relationship between those capabilites and performances. Table 3 shows summary statisctics and indicate that each dimension of capabilites and performances is highly correlated. Hence, we maily focus on the direct relationship between each capability and performance.

Table 3. Summary Statistics

\begin{tabular}{lcccccccc}
\hline & & Mean & S.D. & 1 & 2 & 3 & 4 & 5 \\
\hline 1 & Business capability & 3.24 & 0.99 & & & & & \\
2 & Digital capability & 3.15 & 1.00 & 0.89 & & & & \\
3 & Soft capability & 3.21 & 1.00 & 0.89 & 0.91 & & & \\
4 & Profitability growth & 2.41 & 1.05 & 0.41 & 0.42 & 0.40 & & \\
5 & New product development & 2.34 & 1.01 & 0.38 & 0.39 & 0.37 & 0.90 & \\
6 & New partnership & 2.34 & 1.00 & 0.37 & 0.38 & 0.36 & 0.89 & 0.96 \\
\hline
\end{tabular}

$\mathrm{N}=439$

Source: Own processing

Table 4-7 show the result of OLS regression. As expected, while each capability has significant and positive relationship with each anticipated performance, Table 5 shows interesting results. In case of large firms, no capability has significant impact on new product development. This may indicate that large firms view digital transformation as instrument to renovate business model and network rather than merely developing new product. The findings presented in Table 4-7 does not necessarily mean the impact of those capabilities on three types of performances are confirmed. Rather, it reflects how people in firms perceive or believe the association between capabilities and performances. However, it is still meaningful and valid to understand how various firms interpret encounting situations and changes brought by digital transformation. 
Table 4. OLS regression on firm performances (Small \& Medium Firms)

\begin{tabular}{|c|c|c|c|c|}
\hline \multirow{2}{*}{ Independent variables } & \multicolumn{4}{|c|}{ Profitability } \\
\hline & & \multirow{7}{*}{$\begin{array}{c}0.403 * * * \\
0.050\end{array}$} & & \\
\hline Business capability & $0.417 * * *$ & & & $0.256^{*}$ \\
\hline & 0.050 & & & 0.119 \\
\hline Digital capability & & & & 0.173 \\
\hline & & & & 0.126 \\
\hline \multirow[t]{2}{*}{ Soft capability } & & & $0.392 * * *$ & 0.001 \\
\hline & & & 0.051 & 0.127 \\
\hline \multirow[t]{2}{*}{$N$} & 353 & 353 & 353 & 353 \\
\hline & \multicolumn{4}{|c|}{ New product development } \\
\hline \multicolumn{5}{|l|}{ Independent variables } \\
\hline \multirow{2}{*}{ Business capability } & $0.404 * * *$ & & & 0.244 \\
\hline & 0.049 & & & 0.116 \\
\hline \multirow[t]{2}{*}{ Digital capability } & & $0.387 * * *$ & & 0.129 \\
\hline & & 0.049 & & 0.123 \\
\hline \multirow[t]{2}{*}{ Soft capability } & & & $0.385 * * *$ & 0.051 \\
\hline & & & 0.050 & 0.124 \\
\hline \multirow[t]{2}{*}{$N$} & 353 & 353 & 353 & 353 \\
\hline & \multicolumn{4}{|c|}{ New partnership } \\
\hline \multicolumn{5}{|l|}{ Independent variables } \\
\hline \multirow{2}{*}{ Business capability } & $0.371 * * *$ & & & $0.211+$ \\
\hline & 0.048 & & & 0.114 \\
\hline \multirow[t]{2}{*}{ Digital capability } & & $0.362 * * *$ & & 0.169 \\
\hline & & 0.048 & & 0.121 \\
\hline \multirow[t]{2}{*}{ Soft capability } & & & $0.352 * * *$ & 0.009 \\
\hline & & & 0.049 & 0.123 \\
\hline$N$ & 353 & 353 & 353 & 353 \\
\hline
\end{tabular}

Source: Own processing

Table 5. OLS regression on firm performances (Large Firms)

\begin{tabular}{|c|c|c|c|c|}
\hline Independent variables & \multicolumn{4}{|c|}{ Profitability } \\
\hline \multirow[t]{2}{*}{ Business capability } & $0.291 * *$ & & & -0.569 \\
\hline & 0.107 & & & 0.348 \\
\hline \multirow{2}{*}{ Digital capability } & & $0.386 * * *$ & & $0.679+$ \\
\hline & & 0.109 & & 0.366 \\
\hline \multirow[t]{2}{*}{ Soft capability } & & & $0.332 * *$ & 0.258 \\
\hline & & & 0.102 & 0.371 \\
\hline \multirow[t]{2}{*}{$N$} & 86 & 86 & 86 & 86 \\
\hline & \multicolumn{4}{|c|}{ New product development } \\
\hline \multicolumn{5}{|l|}{ Independent variables } \\
\hline \multirow[t]{2}{*}{ Business capability } & 0.068 & & & -0.50 \\
\hline & 0.101 & & & 0.3361 \\
\hline \multirow[t]{2}{*}{ Digital capability } & & 0.134 & & 0.451 \\
\hline & & 0.105 & & 0.353 \\
\hline \multirow[t]{2}{*}{ Soft capability } & & & 0.106 & 0.178 \\
\hline & & & 0.097 & 0.358 \\
\hline
\end{tabular}




\begin{tabular}{|ccccc|}
\hline $\boldsymbol{N}$ & 86 & 86 & 86 & 86 \\
\hline & \multicolumn{4}{c|}{ New partnership } \\
\cline { 2 - 5 } Independent variables & \multicolumn{3}{c}{} \\
\hline Business capability & 0.122 & & $-0.665+$ \\
& 0.103 & $0.212^{*}$ & & 0.338 \\
Digital capability & & 0.106 & $0.611+$ \\
& & & $0.172+$ & 0.356 \\
Soft capability & & & 0.099 & 0.346 \\
& & 86 & 86 & 86 \\
\hline $\boldsymbol{N}$ & 86 & & & \\
\hline
\end{tabular}

Source: Own processing

Table 6. OLS regression on firm performances (Manufacturing Firms)

\begin{tabular}{|c|c|c|c|c|}
\hline Independent variables & \multicolumn{4}{|c|}{ Profitability } \\
\hline Business capability & $\begin{array}{c}0.388^{* *} \\
0.076\end{array}$ & & & $\begin{array}{l}0.101 \\
0.171\end{array}$ \\
\hline Digital capability & & $\begin{array}{c}0.393 * * * \\
0076\end{array}$ & & $\begin{array}{l}0.021 \\
0.181\end{array}$ \\
\hline Soft capability & & & $\begin{array}{c}0.377 * * * \\
0.073\end{array}$ & $\begin{array}{l}0.268 \\
0.189\end{array}$ \\
\hline \multirow[t]{2}{*}{$N$} & 194 & 194 & 194 & 194 \\
\hline & \multicolumn{4}{|c|}{ New product development } \\
\hline \multicolumn{5}{|l|}{ Independent variables } \\
\hline Business capability & $\begin{array}{c}0.356 * * * \\
0.074\end{array}$ & & & $\begin{array}{l}-0.509 \\
0.336\end{array}$ \\
\hline Digital capability & & $\begin{array}{l}0.352 \\
0.074\end{array}$ & & $\begin{array}{l}0.451 \\
0.353\end{array}$ \\
\hline Soft capability & & & $\begin{array}{l}0.106 \\
0.097\end{array}$ & $\begin{array}{l}0.178 \\
0.358\end{array}$ \\
\hline \multirow[t]{2}{*}{$N$} & 194 & 194 & 194 & 194 \\
\hline & \multicolumn{4}{|c|}{ New partnership } \\
\hline \multicolumn{5}{|l|}{ Independent variables } \\
\hline Business capability & $\begin{array}{l}0.337 \\
0.074\end{array}$ & & & $\begin{array}{l}0.036 \\
0.170\end{array}$ \\
\hline Digital capability & & $\begin{array}{c}0.360^{* * * *} \\
0.074\end{array}$ & & $\begin{array}{l}0.182 \\
0.181\end{array}$ \\
\hline Soft capability & & & $\begin{array}{c}0.356^{* * *} \\
0.073 \\
\end{array}$ & $\begin{array}{l}0.161 \\
0.188\end{array}$ \\
\hline$N$ & 194 & 194 & 194 & 194 \\
\hline
\end{tabular}

Source: Own processing 
Table 7. OLS regression on firm performances (Non-Manufacturing Firms)

\begin{tabular}{|c|c|c|c|c|}
\hline Independent variables & \multicolumn{4}{|c|}{ Profitability } \\
\hline & & \multirow{7}{*}{$\begin{array}{c}0.462^{* * * *} \\
0.056\end{array}$} & & \\
\hline Business capability & $0.461 * *$ & & & 0.23 \\
\hline & 0.058 & & & 0.149 \\
\hline Digital capability & & & & $0.347 *$ \\
\hline & & & & 0.158 \\
\hline \multirow[t]{2}{*}{ Soft capability } & & & $0.427 * * *$ & -0.104 \\
\hline & & & 0.058 & 0.155 \\
\hline \multirow[t]{2}{*}{$N$} & 245 & 245 & 245 & 245 \\
\hline & \multicolumn{4}{|c|}{ New product development } \\
\hline \multicolumn{5}{|l|}{ Independent variables } \\
\hline \multirow[t]{2}{*}{ Business capability } & $0.398 * * *$ & & & 0.206 \\
\hline & 0.056 & & & 0.145 \\
\hline \multirow[t]{2}{*}{ Digital capability } & & $0.401 * * *$ & & $0.324 *$ \\
\hline & & 0.055 & & 0.154 \\
\hline \multirow[t]{2}{*}{ Soft capability } & & & $0.366^{* * *}$ & -0.117 \\
\hline & & & 0.057 & 0.151 \\
\hline \multirow[t]{2}{*}{$N$} & 245 & 245 & 245 & 245 \\
\hline & \multicolumn{4}{|c|}{ New partnership } \\
\hline \multicolumn{5}{|l|}{ Independent variables } \\
\hline \multirow[t]{2}{*}{ Business capability } & $0.385 * * *$ & & & 0.197 \\
\hline & 0.055 & & & 0.144 \\
\hline \multirow[t]{2}{*}{ Digital capability } & & $0.386 * * *$ & & $0.283+$ \\
\hline & & 0.054 & & 0.153 \\
\hline \multirow{2}{*}{ Soft capability } & & & $0.357 * * *$ & -0.081 \\
\hline & & & 0.056 & 0.149 \\
\hline$N$ & 245 & 245 & 245 & 245 \\
\hline
\end{tabular}

Source: Own processing

We coduct additional analysis to explore more detailed picture of how firm size and industry type conjontly influence the relationship between capabilities and expected performance. While most of main effect of individual capability show significant and positive effect of each expected performance, full model illustrate more interesting findings. (We do not report the main effect of individual capability here due to the space limit). Table 8 shows that large-manufacturing firms and smal-medium non-manufacturing firms demonstrate some statistically signifianct results. Interestingly, in case of large-manufacturing firms, business capabiliy and firm profitability are negatively related. This finding does not mean business capability for digital transformation leads firms experience to lower profitability. Instead it implies that people in large-manufacturing firms does not view business capability as a key determinant of profitability in the era of digital transformation. However, they consider digital capability as a positive and key determinant of it. 
Table 8. OLS regression on three dimensions of firm performance

\begin{tabular}{|c|c|c|c|c|}
\hline \multirow[b]{2}{*}{$\begin{array}{c}\text { Independent } \\
\text { variables }\end{array}$} & \multicolumn{4}{|c|}{ Profitability } \\
\hline & $\begin{array}{l}\text { SMEs } \\
\text { MFG }\end{array}$ & $\begin{array}{l}\text { Large } \\
\text { MFG }\end{array}$ & $\begin{array}{c}\text { SMEs } \\
\text { non-MFG }\end{array}$ & $\begin{array}{c}\text { Large } \\
\text { non-MFG }\end{array}$ \\
\hline Business capability & $\begin{array}{c}0.229 \\
(0.182)\end{array}$ & $\begin{array}{c}-1.257 * * \\
(0.446)\end{array}$ & $\begin{array}{c}0.260 \\
(0.160)\end{array}$ & $\begin{array}{l}-0.0365 \\
(0.497)\end{array}$ \\
\hline Digital capability & $\begin{array}{l}-0.023 \\
(0.198)\end{array}$ & $\begin{array}{l}1.000^{*} \\
(0.401)\end{array}$ & $\begin{array}{l}0.303+ \\
(0.166)\end{array}$ & $\begin{array}{c}0.456 \\
(0.882)\end{array}$ \\
\hline Soft capability & $\begin{array}{c}0.210 \\
(0.212)\end{array}$ & $\begin{array}{c}0.500 \\
(0.363)\end{array}$ & $\begin{array}{l}-0.115 \\
(0.161)\end{array}$ & $\begin{array}{c}0.030 \\
(0.993)\end{array}$ \\
\hline \multirow[t]{2}{*}{$N$} & 160 & 34 & 193 & 52 \\
\hline & \multicolumn{4}{|c|}{ New product development } \\
\hline $\begin{array}{l}\text { Independent } \\
\text { variables }\end{array}$ & $\begin{array}{l}\text { SMEs } \\
\text { MFG }\end{array}$ & $\begin{array}{l}\text { Large } \\
\text { MFG }\end{array}$ & $\begin{array}{c}\text { SMEs } \\
\text { non-MFG }\end{array}$ & $\begin{array}{c}\text { Large } \\
\text { non-MFG }\end{array}$ \\
\hline Business capability & $\begin{array}{c}0.178 \\
(0.178)\end{array}$ & $\begin{array}{l}-0.642 \\
(0.437)\end{array}$ & $\begin{array}{l}0.270+ \\
(0.153)\end{array}$ & $\begin{array}{l}-0.495 \\
(0.488)\end{array}$ \\
\hline Digital capability & $\begin{array}{l}-0.114 \\
(0.195)\end{array}$ & $\begin{array}{c}0.628 \\
(0.393)\end{array}$ & $\begin{array}{l}0.280+ \\
(0.159)\end{array}$ & $\begin{array}{l}-0.105 \\
(0.865)\end{array}$ \\
\hline Soft capability & $\begin{array}{l}0.347+ \\
(0.208)\end{array}$ & $\begin{array}{c}0.093 \\
(0.356)\end{array}$ & $\begin{array}{l}-0.126 \\
(0.154)\end{array}$ & $\begin{array}{c}0.737 \\
(0.975)\end{array}$ \\
\hline $\boldsymbol{N}$ & 160 & 34 & 193 & 52 \\
\hline \multirow[b]{2}{*}{$\begin{array}{l}\text { Independent } \\
\text { variables }\end{array}$} & \multicolumn{4}{|c|}{ New partnership } \\
\hline & $\begin{array}{l}\text { SMEs } \\
\text { MFG }\end{array}$ & $\begin{array}{l}\text { Large } \\
\text { MFG }\end{array}$ & $\begin{array}{c}\text { SMEs } \\
\text { non-MFG }\end{array}$ & $\begin{array}{c}\text { Large } \\
\text { non-MFG }\end{array}$ \\
\hline Business capability & $\begin{array}{c}0.171 \\
(0.175)\end{array}$ & $\begin{array}{c}-1.423 * * \\
(0.453)\end{array}$ & $\begin{array}{c}0.230 \\
(0.154)\end{array}$ & $\begin{array}{l}-0.137 \\
(0.471)\end{array}$ \\
\hline Digital capability & $\begin{array}{c}0.046 \\
(0.191)\end{array}$ & $\begin{array}{c}1.172^{* *} \\
(0.407)\end{array}$ & $\begin{array}{c}0.249 \\
(0.160)\end{array}$ & $\begin{array}{l}-0.0132 \\
(0.836)\end{array}$ \\
\hline Soft capability & $\begin{array}{c}0.161 \\
(0.204)\end{array}$ & $\begin{array}{c}0.457 \\
(0.369) \\
\end{array}$ & $\begin{array}{r}-0.0814 \\
(0.155) \\
\end{array}$ & $\begin{array}{c}0.342 \\
(0.941) \\
\end{array}$ \\
\hline$N$ & 160 & 34 & 193 & 52 \\
\hline
\end{tabular}

Source: Own processing

\section{Discussion}

Our study provide unique insights in several ways. First, we offer useful concepts to evaluate capabilities for and expected outcome of digital transformation. In our survey, three important capabilities for digital transformation (business, digital, soft capabilities) and three expeceted performanace improvement (profitability, new product development, new partnership) are addressed. More importantly, our study demonstrate how those concepts are differently perceived to an individual firm. While large size firms and manufacturing firms show more positive perspective on digital transformation in terms of both capabilities and expected outcomes, those firms demonstrate some interesting patterns such as negative or no effect (in fact, perceived expectation) of businss capability on performances. 
More importantly and interestingly, by investigating firm size effect and industry effect, our study offer a clue for 'digital divide' that can potentially threaten the survival of numerous firms that fail to adopt relevant skills and capabilities to prepare digital transformation. As digital transformation will come with unprecedented degree of convergence across industrial sectors, the negative effect of digital divide can creat trenmendous ineuqlity among firms (e.g., large firm vs. small firm). This may recall for policy maker considering some policy efforts for balanced development.

\section{References}

1. L. Li, F. Su, W. Zhang, JY. Mao, Digital Transformation by SME Entrepreneurs: A Capability Perspective. Information Systems Journal, 28(6), 1129-1157 (2018)

2. R. Eller, P. Alford, A. Kallmünzer, M. Peters, M. Antecedents, Consequences, and Challenges of Small and Medium-Sized Enterprise Digitalization. Journal of Business Research, 112, 119-127 (2020)

3. E. Siachou, D. Vrontis, E. Trichina, Can Traditional Organizations Be Digitally Transformed By Themselves? The Moderating Role of Absorptive Capacity and Strategic Interdependence. Journal of Business Research, 124, 408-421 (2021)

4. M. Savastano, C. Amendola, F. Bellini, F. D'Ascenzo, Contextual Impacts on Industrial Processes Brought by the Digital Transformation of Manufacturing: A Systematic Review. Sustainability, 11(3), 891-929 (2019)

5. K. Vogelsang, K. Liere-Netheler, S. Packmohr, S, U. Hoppe, Success Factors for Fostering a Digital Transformation in Manufacturing Companies. Journal of Enterprise Transformation, 8(1-2), 121-142 (2018)

6. T. von Leipzig, M. Gamp, M, D. Manz, K. Schöttle, P. Ohlhausen, G Oosthuizen, D. Palm, K. von Leipzig, Initialising Customer-Orientated Digital Transformation in Enterprises. Procedia Manufacturing. 8, 517-524 (2017)

7. ML. Martín-Peña, JM. Sánchez-López, E. Díaz-Garrido, Servitization and Digitalization in Manufacturing: The Influence on Firm Performance. Journal of Business \& Industrial Marketing, 35(3), 564-574 (2020)

8. S. Ribeiro-Navarrete, D. Botella-Carrubi, D. Palacios-Marqués, M. Orero-Blat, The Effect of Digitalization on Business Performance: An Applied Study of KIBS. Journal of Business Research, 126, 319-326 (2021)

9. Y. Luo. New OLI advantages in digital globalization. International Business Review, 30(2) (2021)

10. J. Oh, S. Rhee, The influence of supplier capabilities and technology uncertainty on manufacturer supplier collaboration: a study of the Korean automotive industry. International Journal of Operations \& Production Management, 28(6), 490-517 (2008)

11. DJ. Teece, Profiting from technological innovation: Implications for integration, collaboration, licensing and public policy. Research Policy, 15(6), 285-305 (1986)

12. DJ. Teece, Profiting from innovation in the digital economy: standards, complementary assets, and business models in the wireless world. Research Policy, 47(8), 1367-1387 (2018) 